\title{
Clinical implications of temperature curve complexity in critically ill patients
}

\author{
Manuel Varela, MD, PhD; Marta Calvo, MD; Miriam Chana, MD; Ivan Gomez-Mestre, PhD; \\ Rosa Asensio, MD; Pedro Galdos, MD
}

Objective: In certain physiologic systems, disease is associated with a loss of complexity in system's output. We test the hypothesis that, in critically ill patients, there is an inverse relation between the complexity of the temperature curve and the clinical status. We also consider whether complexity analysis of the temperature curve may have prognostic value.

Design: Prospective, observational study.

Setting: Intensive care unit of a general hospital in Madrid, Spain.

Patients: Twenty-four successive patients admitted in the intensive care unit with multiple organ failure.

Interventions: Skin temperature was measured every 10 mins from inclusion in the study until discharge or death (median length of stay 18.8 days, interquartile range 86 ).

Measurements: From the temperature time series, hourly approximate entropy measurements were obtained. Clinical status was evaluated using the Sequential Organ Failure Assessment (SOFA) score.

Main Results: A significant inverse relationship between ap- proximate entropy and the attributed SOFA score was observed in $89 \%$ of the patients considered. Both mean and minimum approximate entropy were significantly lower in patients who died than in patients who survived (mean approximate entropy, 0.47 vs. 0.61 ; minimum approximate entropy, 0.24 vs. 0.40 ; in both cases $p<.001)$. To evaluate the prognostic value of both mean and minimum approximate entropy, we fitted logistic regression models against survival. An increase in 0.1 units in minimum or mean approximate entropy increased 15.4- and 18.5-fold the odds of surviving, respectively.

Conclusions: The clinical status of patients suffering multiple organ failure is inversely correlated to the complexity of the temperature curve expressed as approximate entropy. Reduced complexity has dismal prognostic implications. Its assessment is noninvasive and inexpensive and allows for real-time continuous monitoring of clinical status.

KEY WoRDS: body temperature; multiple organ failure; complexity analysis; nonlinear dynamics; approximate entropy; Sequential Organ Failure Assessment
M easurement of body temperature is one of the oldest clinical tools available, and fever still remains a reliable indicator of illness. On the other hand, the prognostic and diagnostic value of fever is quite limited. Generally, if a patient is running a fever, he or she is most likely sick (although this does not tell us much more about the etiology). When a patient does not have a fever, body temperature does not furnish any clinical information. In any case, a patient cannot be said to be more seriously

From Servicio de Medicina Interna (MV, MCa) and Unidad de Cuidados Intensivos (MCh, RA, PG), Hospital de Móstoles, Madrid, Spain; and Department of Biology, Boston University, Boston, MA (IG-M).

Supported by grant PI 030591 of the Fondo de Investigacion Sanitaria, Ministerio de Sanidad y Consumo, Spain.

The authors have no financial interest to disclose. ill because she has a higher temperature, nor can she be considered to be healthy because she does not have a fever.

Theoretically, temperature is a quantitative variable, but in actual clinical practice it behaves like a dichotomous variable (febrile/afebrile), with a poorly defined cutoff point (1). Attempts to consider fever as a continuous quantitative variable have been largely unsuccessful, and fever categories (e.g., intermittent, continuous) have had little clinical success. Nevertheless, in patients both with or without fever, temperature is the end result of a finely tuned, complex system and might afford a window on significant physiologic information.

An approach that would allow temperature (or some other measure obtained from it) to be used as a continuous, nondichotomous variable having physiologic significance could be a novel tool potentially useful in clinical settings. Furthermore, it could also be used for afebrile patients, for whom classic fever measurement is of no help.
Certain methods derived from nonlinear dynamics and complexity analysis could be put to use for this purpose. Varela et al. (2) reported that in healthy subjects, the temperature curve behaves like a natural fractal whose complexity may be analyzed in a consistent manner. In addition, they observed that complexity decreased significantly with age.

The present article is an attempt to extend that approach to critically ill patients. The hypothesis tested was that in severely ill patients, the thermoregulatory system will display this damage as a deterioration of its output, manifested as a decrease in the complexity of the temperature curve (irrespective of whether fever is present). Approximate entropy (ApEn), a well-established measure of complexity in time series, was used for this purpose. This variable has been shown to be robust and insensitive to baseline fluctuations (3-7).

Our purpose was to investigate the complexity of the temperature curve in 
critically ill patients. Namely, we searched to establish the following:

1. In each individual patient, whether there is a correlation between the time trend for clinical status as measured by the Sequential Organ Failure Assessment (SOFA) score and complexity in the recorded temperature values

2. In the whole sample, whether the complexity measurements have prognostic value as indicators of the likelihood of patient survival

\section{MATERIALS AND METHODS}

Patients. The study was carried out in the intensive care unit (ICU) at the Hospital de Mostoles, in one of the outlying areas around Madrid, Spain. The study population consisted of 24 consecutive patients diagnosed with multiple organ failure. The study spanned from January 2002 to March 2004 but was interrupted from June 2003 to January 2004 because of technical problems (no data logger or transducer available).

Multiple organ failure was defined as two or more impaired organs (respiratory, cardiovascular, coagulation, central nervous system, liver, or renal), irrespective of the primary diagnosis or cause of admission.

The patients were 13 women (mean age, 62.4; range, 18-84) and 11 men (mean age; 58.0, range; 36-81). Informed consent was obtained from the patient or a family member whenever possible. In three cases, the patients died before informed consent could be obtained. However, since consent had not been refused (it had proved impossible to contact a family member) and since temperature measurement is a routine form of clinical monitoring that can be regarded as being more of an observational register than a form of intervention, it was decided to include these three patients. The study was approved by the hospital's Ethics and Research Review Board.

Table 1 summarizes the clinical data for the patients.

Temperature Measurement. A thermistor temperature sensor (Datalogger Spectrum 1000, Veriteq Instruments, Richmond, BC, Canada) was attached to the right or left hypochondrium (upper abdomen) of the patients included in the study, and temperature readings were taken every 10 mins from inclusion until discharge from the ICU or death.

The readings yielded successive series of 30 consecutive hours (180 readings) each with a 1-hr offset and 29 hrs of overlap between every two adjacent series. These series were used to calculate the ApEn values for each successive time period. Accordingly, the method produced one ApEn value per hour from the day after admission, with each value encompassing the preceding $30 \mathrm{hrs}$, until the patient's discharge or death. To avert the influence of pre- or perimortem conditions, the last four temperature readings preceding discharge or death were not included. Stationarity was verified by means of the reverse arrangements test (8).

Patients were occasionally disconnected (for radiologic examinations, for surgical procedures, or by accident). Furthermore, accidental disconnection was assumed to have occurred when there was a difference of $>3^{\circ} \mathrm{C}$ between any two consecutive readings or when the temperature reading was $<30^{\circ} \mathrm{C}$. Where the disconnection spanned one or two readings, the value was calculated by interpolation based on the preceding and the following reading. Where the disconnection lasted more than three readings, the series was stopped and restarted from the beginning (meaning that no complexity value was available until 30 hrs later).

Globally, complexity values were obtained for an average of $78.5 \%$ (range, $40-100 \%$ ) of the total possible number of hours for each patient (not counting the first $30 \mathrm{hrs}$ ).

Sequential Organ Failure Assessment (SOFA). The extent of organ dysfunction was measured in all the patients every 48 hrs using the SOFA scoring system $(9,10)$. The SOFA score is a widely accepted tool for assessing severity and morbidity based on temporal measurement of organ dysfunction. It has been shown to correlate well with mortality rate (11) and it is considered as accurate as the other systems available, namely the Multiple Organ Dysfunction Score (12) and Logistic Organ Dysfunction scoring (13).

SOFA analyzes the degree of physiologic impairment of various organs or systems (respiratory system, hemodynamic system, coagulation, renal function, liver function, and level of consciousness), with scoring running from 0 (no organ dysfunction) to 24 (maximum organ dysfunction) (Table 2). Hourly SOFA scoring is impossible for practical reasons (it requires an arterial blood sample and would be hardly justifiable on clinical grounds), and consequently hourly SOFA scores, termed attributed scores, were calculated by interpolation from the empirical values compiled every 48 hrs, assuming a linear rate of increase.

Complexity Analysis: ApEn. ApEn is a measure of time series complexity. Given a time series, three variables are needed to measure ApEn, namely, $\mathrm{m}, \mathrm{r}$, and $\mathrm{N}-\mathrm{m}$ being the length of the vectors of the curve to be compared (usually, $m=2$, i.e., the vectors to be compared are composed of two successive points); $r$ being the range defining two measurements as similar (a value of between 0.15 and 0.20 standard deviations normally being used); and $\mathrm{N}$ being the total number of measurements considered (there being a consensus that a value $>10^{\mathrm{m}}$ is needed).

By way of illustration, let us suppose we have a time series of $\mathrm{N}$ temperature measurements (Fig. 1). Let us take the vector consist- ing of the first pair of successive data points, $\mathrm{p}_{1}$ and $\mathrm{p}_{2}$, from the total series of $\mathrm{N}$ points. We then seek all the vectors consisting of points $\mathrm{p}_{\mathrm{i}}$ and $\mathrm{p}_{\mathrm{i}+1}$ in the series that fulfill the conditions:

$$
\begin{aligned}
& \left(\mathrm{p}_{1}-\mathrm{r}\right)<\mathrm{p}_{\mathrm{i}}<\left(\mathrm{p}_{1}+\mathrm{r}\right) \text { and } \\
& \qquad\left(\mathrm{p}_{2}-\mathrm{r}\right)<\mathrm{p}_{\mathrm{i}+1}<\left(\mathrm{p}_{2}+\mathrm{r}\right)
\end{aligned}
$$

This selects all the vectors $\left[p_{i}, p_{i}+{ }_{1}\right]$ similar to $\left[\mathrm{p}_{1}, \mathrm{p}_{2}\right]$ (i.e., whose origin is in the range of $\mathrm{p}_{1} \leq \mathrm{r}$ and whose end point is in the range $p_{2}$ ! $r$ ). We next find which proportion of these vectors is followed by a value of $\mathrm{p}_{\mathrm{i}}+2$ that falls within the range of $\left(p_{3}-r\right)<p_{i}+2<\left(p_{3}+r\right)$. This is a measure of the extent to which a vector similar to $\left[\mathrm{p}_{1}, \mathrm{p}_{2}\right]$ will condition a subsequent point similar to $\mathrm{p}_{3}$. The procedure is repeated for all successive pairs of points $\left[\mathrm{p}_{1}, \mathrm{p}_{2}\right],\left[\mathrm{p}_{2}, \mathrm{p}_{3}\right], \ldots,\left[\mathrm{p}_{\mathrm{n}-1}, \mathrm{p}_{\mathrm{n}}\right]$, in each case measuring the conditional probability that, given a vector similar to the vector with which it is being compared, the next point will be similar to the point following the pattern vector.

ApEn is the average logarithm of the conditional probability for each pair of points, after the sign has been changed to ensure a positive value. Thus, ApEn provides an inverse measure of the extent to which knowledge of any two successive points allows the next to be predicted. The measure will be higher the greater the irregularity of the series of data points, reaching a maximum value for an entirely random series.

In the case of our series, $m=2$ (i.e., the vectors to be compared consisted of each pair of successive readings), $r=0.2$ SD for the time series being analyzed, and $n=180$ (i.e., 180 readings were analyzed, one reading every 10 mins for $30 \mathrm{hrs}$ ).

The program used to calculate ApEn was written in Python (http://www.python.org) and is available from the author on request (mvarela.hmtl@salud. madrid.org).

As an example, Figure 2 shows two real temperature series from our patients, one with high and one with low ApEn values.

Statistics. Only patients with at least three empirical SOFA measurements (and hence a length of stay of Š5 days) were analyzed to test for within-individual temporal correlation between ApEn and SOFA because we believed that less than three empirical measures would make correlation meaningless. Consequently, patients $3,7,18,19$, and 23 were dropped out for this analysis, reducing the sample size to 19.

All 24 patients were subsequently included for all other analyses. Linear regression was 
Table 1. Summary of clinical information and within-individual association between approximate entropy (ApEn) and Sequential Organ Failure Assessment (SOFA)

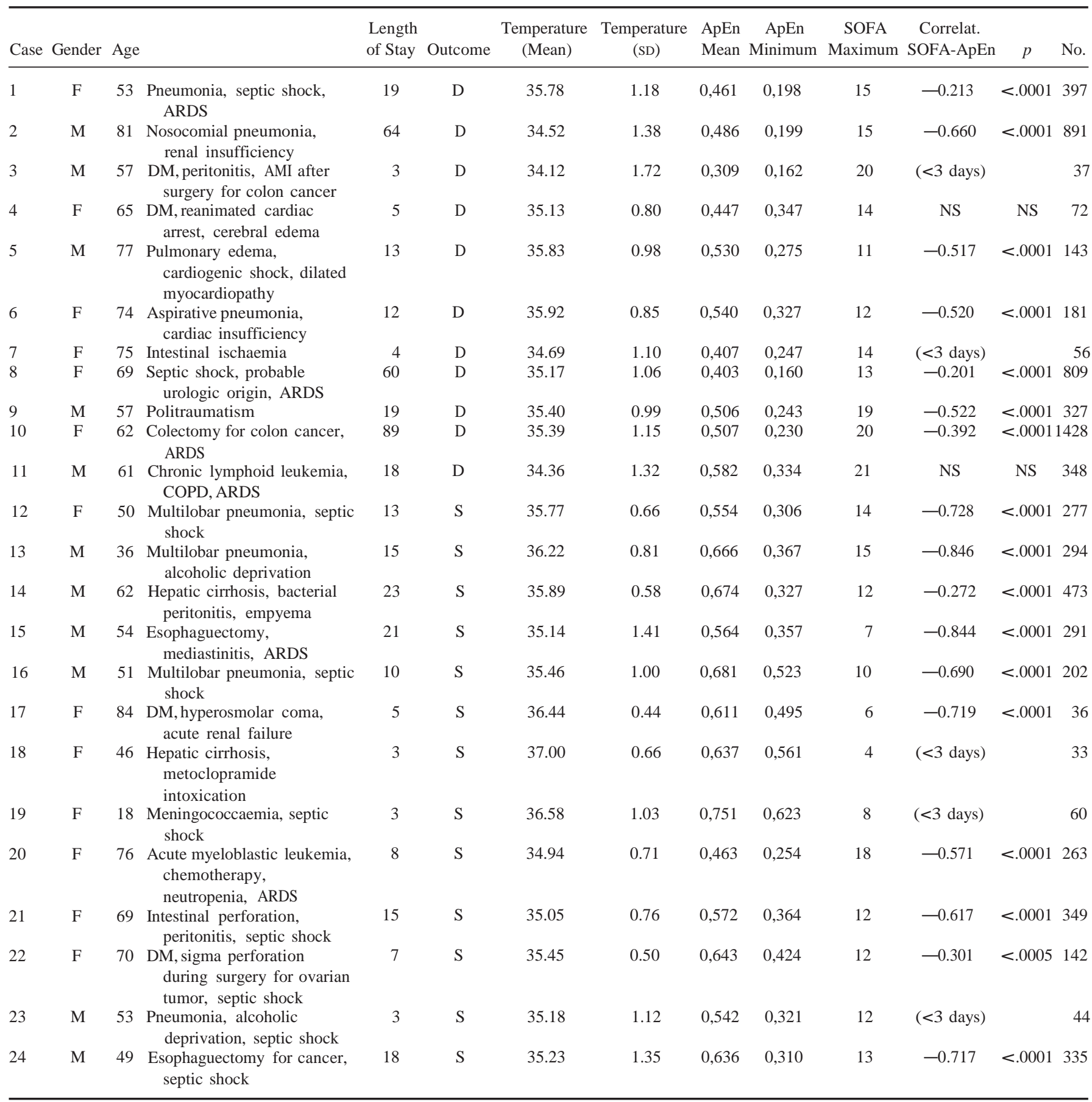

ApEn mean, mean value of $\operatorname{ApEn}_{(2,0.2 \mathrm{SD}, 180)}$ for the temperature series; ApEn minim, minimum value of $\operatorname{ApEn}_{(2,0.2 \mathrm{SD}, 180)}$ for the temperature series; SOFA max, maximum value of SOFA attained by the patient; Correlat SOFA-ApEn, correlation coefficient for lineal correlation between hourly ApEn and attributed hourly SOFA scores (patients admitted for $<3$ days were not included in the analysis); N, number of hours for which there was an ApEn measured; ARDS, acute respiratory distress syndrome; DM, diabetes mellitus; AMI, acute myocardial infarction; COPD, chronic obstructive pulmonary disease; D, died; S, survived.

used to test the association between complexity of the temperature curve and clinical condition, as represented by the attributed SOFA score in each patient. Linear regression was also used to analyze the relationship between minimum ApEn and maximum SOFA and between mean ApEn and mean SOFA in the whole population. Analysis of variance was used to examine the relationship between complexity (minimum and mean) and survival in the entire group.

To adjust for the effect of age, we fitted a linear regression between age and either mean or minimum ApEn. The residuals from these regressions were then subsequently regressed against SOFA to obtain the "age-free" relation between ApEn and SOFA. Similarly, we regressed these residuals against survival to obtain the ageadjusted relation between ApEn and survival. 
Sequential Organ Failure Assessment (SOFA)

\begin{tabular}{|c|c|c|c|c|}
\hline Points & 1 & 2 & 3 & 4 \\
\hline $\begin{array}{l}\text { Pulmonary, } \mathrm{PaO}_{2} / \mathrm{FIO}_{2} \text {, } \\
\text { mm Hg }\end{array}$ & $<400$ & $<300$ & $<200$ & $<100$ \\
\hline Liver, bilirubin, mg/dL & $1.2-1.9$ & $2.0-5.9$ & $6.0-1.9$ & $>12.0$ \\
\hline $\begin{array}{l}\text { Cardiovascular, } \\
\text { hypotension, } \\
\text { adrenergic drugs, } \\
\mu \mathrm{g} / \mathrm{kg} / \mathrm{min}\end{array}$ & MAP, >70 mm Hg & $\begin{array}{c}\text { Dopamine, 5, or } \\
\text { dobutamine, any dose }\end{array}$ & $\begin{array}{c}\text { Dopamine, }>5 \text {, or } \\
\text { epinephrine, }<0.1 \text {, or } \\
\text { norepinephrine, }<0.1\end{array}$ & $\begin{array}{c}\text { Dopamine, }>15 \text {, or } \\
\text { epinephrine, }>0.1 \text {, or } \\
\text { norepinephrine, }>0.1\end{array}$ \\
\hline $\begin{array}{l}\text { Central nervous system, } \\
\text { Glasgow Coma Scale }\end{array}$ & $13-14$ & $10-12$ & $6-9$ & $<6$ \\
\hline $\begin{array}{l}\text { Renal, creatinine, mg/ } \\
\text { dL (or urine output, } \\
\text { mL) }\end{array}$ & $1.2-1.9$ & $2.0-3.4$ & $3.5-4.9$ (<500 mL/day) & $>5.0(<200 \mathrm{~mL} /$ day $)$ \\
\hline
\end{tabular}

MAP, mean arterial pressure.

Finally, a maximum likelihood approach was used to fit a logistic regression equation to the data to predict the probability of patient survival based on either the minimum ApEn value or the mean ApEn value.

All statistics were performed on SPSS 11.0.1 software (SPSS, Chicago, IL).

\section{RESULTS}

Mean patient age was 63 yrs (range, 18-84). ICU stay length ranged from 3 to 89 days with a mean length of $18.8 \mathrm{~d}$.

There were no significant differences between genders with respect to age, ICU stay length, SOFA score, or mortality. Patients who died tended to be older than those who survived (66.4 vs. 55.2 yrs), but this tendency did not reach statistical significance $(p=.069)$.

As expected, mean and maximum SOFA scores in patients who died differed significantly from those in patients who survived (mean SOFA score 11.04 [sD 2.68] vs. 5.66 [SD 2.68], $\mathrm{F}_{1,22}=17.77, p<.001$ ) and maximum SOFA score 15.8 [SD 3.54] vs. 11.0 [sD 3.89], $\mathrm{F}_{1,22}=9.89, p<.005$ ).

Therapeutic efforts were curtailed for certain patients (cases 5, 12, and 13) at the request of the families, and in one of those cases (patient 5) the patient was discharged to a general internal medicine ward so that he could die in the company of his family. Death took place 4 days after discharge from the ICU. The patient was discharged alive from the ICU, so for purposes of the analysis he was classified as survivor.

Temperature Measurements. There was a small but significant difference in the mean temperature and SD between

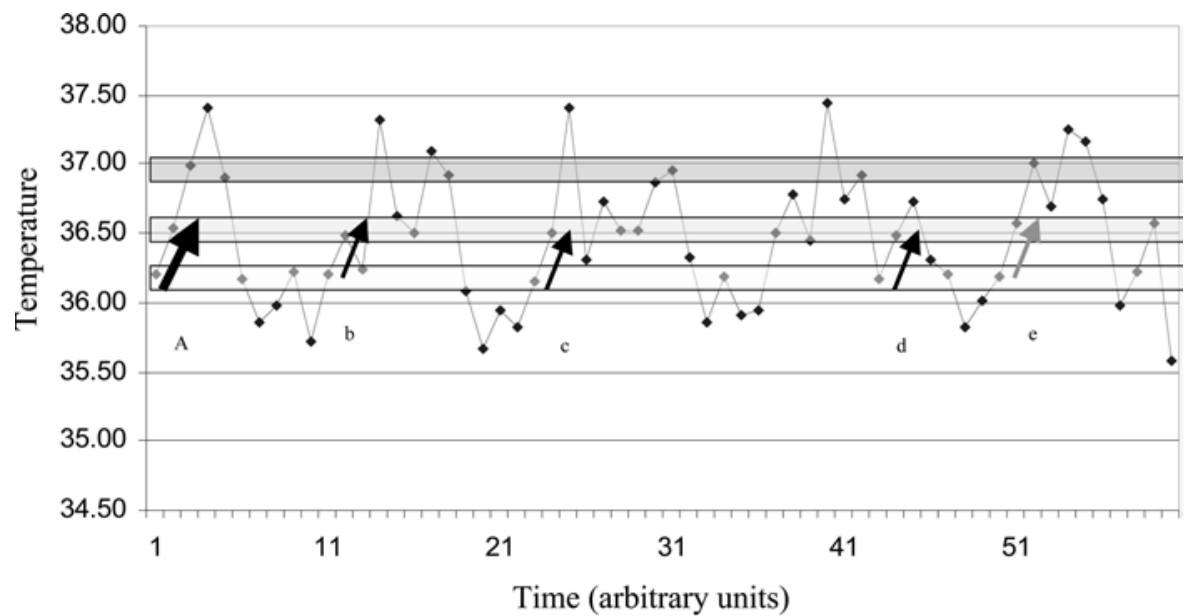

$\square p_{2} \pm r$

$p_{3} \pm r$

Figure 1. Approximate entropy determination. Estimation of the conditional probability for a first vector $\mathrm{p}_{1}-\mathrm{p}_{2}(A)$. The plot shown has only four vectors similar to $A$ (i.e., having the origin within the range of $\mathrm{p}_{1} \leq \mathrm{r}$ and the end point within the range of $\left.\mathrm{p}_{2} \leq \mathrm{r}\right)$. Of these, only one $(e)$ is followed by a point within the range of $\mathrm{p}_{3}$ 】 r. Accordingly, the conditional probability would be $1: 4=0.25$. The conditional probability is calculated in the same manner for all pairs of points $\mathrm{p}_{1}-\mathrm{p}_{2}, \mathrm{p}_{2}-\mathrm{p}_{3} \ldots \mathrm{p}_{\mathrm{n}-1}-\mathrm{p}_{\mathrm{n}}$. ApEn is the mean of the logarithm of all the conditional probabilities after changing the sign.

survivors and nonsurvivors (mean temperature 35.72 vs. 35.12 ; sD 0.84 vs. 1.14 , $p<.05$ in both cases). Temperature did not differ significantly between genders. There was a trend toward lower mean temperature with advanced age that did not reach statistical significance $(r=$ -.38, $p=.064)$.

Complexity of the Temperature Curve. There were no significant differences in ApEn values between male and female patients. Significant negative correlations between age and ApEn values were found for both the mean ApEn ( $\mathrm{r}=$
-.48, $p<.05)$ and the minimum ApEn ( $\mathrm{r}$ $=-.44, p<.05$ ).

Within-Individual Correlation Between ApEn and SOFA Score. ICU stay length was Š5 days in 19 of the 24 patients; hence, three empirical SOFA determinations could be made in all those patients. In 17 of these 19 patients (89\%), there was a significant inverse correlation between the ApEn values and the attributed SOFA scores $(p<.001$ for the 17 cases). In the other two cases, the relationship did not attain statistical significance. 


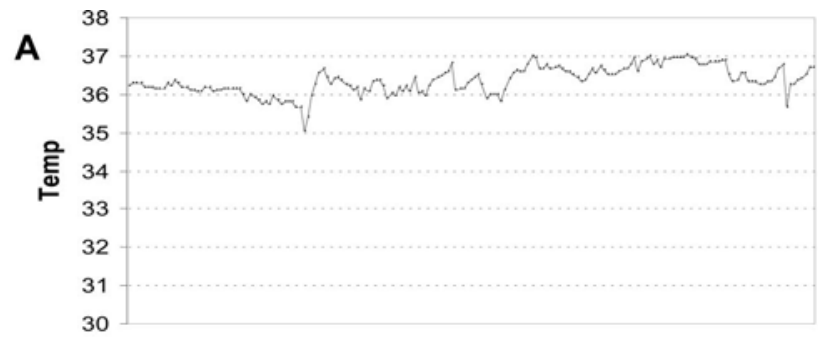

Patient 12

$\operatorname{ApEn}_{(2,0.2 \mathrm{SD}, 180)}=0.834$

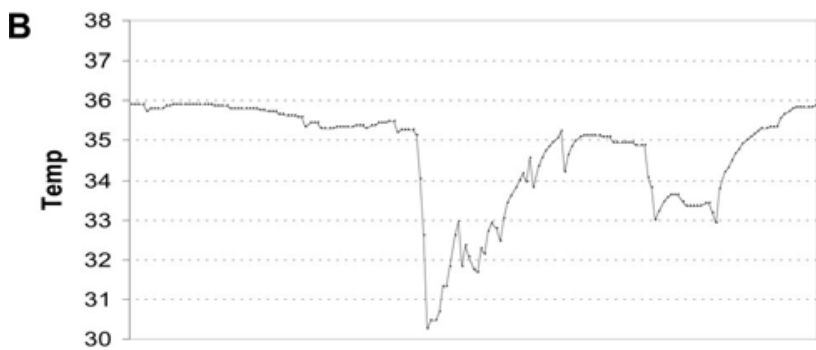

Patient 7

$\operatorname{ApEn}_{(2,0.2 \mathrm{SD}, 180)}=0.25 \mathrm{~g}$

Time (30 hours)

Figure 2. Examples of temperature tracings. Two examples of 30-hr-long temperature series, one showing high complexity $(A)$ and the other showing low complexity (B). ApEn, approximate entropy.

Figure 3 shows SOFA and ApEn curves from a survivor and a nonsurvivor. Figure 4 displays the initial and final ApEn of each patient.

Among-Individual Correlation Between ApEn and SOFA Score. The relationship between minimum ApEn and maximum SOFA attained by each patient was also analyzed in the group as a whole, including all 24 patients in this analysis. A significant inverse correlation was observed $(\mathrm{r}=-.708, p<.001)$ between minimum ApEn and maximum SOFA. Likewise, there was a significant inverse correlation between mean ApEn and mean SOFA ( $r=-.731, p<.001)$. These correlations remained significant after adjustment for the effect of age (minimum ApEn, $\mathrm{r}=-.718, p<.001$; mean ApEn, $\mathrm{r}=-.727, p<.001$ )

Relationship Between ApEn and Survival. The mean and minimum ApEn for patients who died were significantly lower than for those who lived (minimum ApEn, 0.24 [sD 0.07] vs. 0.40 [sD 0.11)], $p$ $<$.001; mean ApEn, 0.47 [SD 0.08] vs. 0.61 [SD 0.07], $p<.001$ ) (Fig. 5). The differences remained significant even after adjusting for the effect of age.

The relative risk of dying decreased gradually in the different terciles both for the minimum ApEn and mean ApEn (0.87 in the lowest tercile, 0.50 in the middle tercile, and 0 in the highest ter- cile for both minimum and mean ApEn). When we set a minimum ApEn value of 0.28 as a cutoff point, the test was able to discriminate between the patients who died and the patients who survived with a sensitivity of 0.73 (95\% confidence interval [CI], $0.39-0.93)$, a specificity of 0.93 (95\% CI, 0.62-1.00), a positive predictive power of 0.89 (95\% CI, 0.51-0.99), and a negative predictive power of 0.80 (95\% CI, 0.51-0.95). The discriminating ability of the mean ApEn value was similar; for example, a cutoff point of 0.55 offered a sensitivity of 0.91 (95\% CI, 0.57-0.99), a specificity of 0.85 (95\% CI, 0.54-0.97), a positive predictive power of 0.83 (95\% CI, 0.51-0.97), and a negative predictive power of 0.92 (95\% CI, 0.60-1.0).

The logistic regression equation for predicting death based on the minimum ApEn value turned out to be significant $\left(\mathrm{y}^{2}=14.99, p=.0001, \mathrm{R}^{2}\right.$ Nagelkerke $=$ $.621)$. With a cutoff point probability of 0.5 , the model classified correctly $83.3 \%$ of the patients. The coefficients obtained were constant $=8.31(\mathrm{sE}=3.66)$ and $\mathrm{H}$ $=-27.36(\mathrm{sE}=11.81)$.

A significant logistic regression equation was also constructed based on the mean ApEn value $\left(\mathrm{y}^{2}=16.44, p<\right.$ $00001, \mathrm{R}^{2}$ Nagelkerke $\left.=.675\right)$. The equation classified correctly $87.0 \%$ of the patients. The coefficients obtained were constant $=16.0(\mathrm{SE}=7.0)$ and $\mathrm{H}=$ -29.86 ( $\mathrm{sE}=12.39)$.

Thus, the probability of dying was

$$
p=\frac{\mathrm{e}^{(8.31-[27.36 \cdot \text { minimum ApEn }])}}{1+\mathrm{e}^{(8.31-[27.36 \cdot \text { minimum ApEn }])}}
$$

or

$$
p=\frac{\mathrm{e}^{(16-[29.86 \cdot \text { mean ApEn] })}}{1+e^{(16-[29.86 \cdot \text { mean ApEn }])}}
$$

An increase in 0.1 units in the minimum or mean ApEn increased 15.4- and 18.5-fold the odds of surviving, respectively.

In both cases, the equations remained significant after correcting for the effect of age on ApEn. Actually, most of the effect of age was gathered by ApEn. A logistic regression model with age as the only factor had a -2logLikelihood $=29.271$, with Wald $_{\mathrm{age}}=2.955$. When minimum ApEn was introduced in the model, -2logLikelihood became 16.224, Wald age $_{1}=1.555$, and Wald $_{\mathrm{ApEn}}=5.525$. Similar results were obtained when using mean ApEn (-2logLikelihood 16.341, Wald ${ }_{\text {age }}=0.316$, and Wald $_{\mathrm{ApEn}}=5.347$ ).

\section{DISCUSSION}

The complexity of the temperature curve is tightly inversely correlated with the severity of the patient's condition. Both mean and minimum ApEn were significantly lower in patients who died than in patients who survived.

Admittedly, the differences in temperature complexity between survivors and nonsurvivors could be biased by other issues related to the clinical status (e.g., agitation, disconnections). Nevertheless, no correlation could be demonstrated between SOFA or ApEn and the percentage of hours from which a complexity value could be obtained in each patient. This suggests that the influence of clinical status on the recording process is not substantial and that there is no bias toward preferentially recording patients in a better (or worse) clinical status.

Another possible limitation of our study stems from the fact that most of the attributed hourly SOFA scores are not proper empirical data but are interpolations between real measurements. However, more frequent systematic determinations were considered unethical, and we thought that this "attributed SOFA score" was the best possible approximation to a continuous evaluation of clinical 

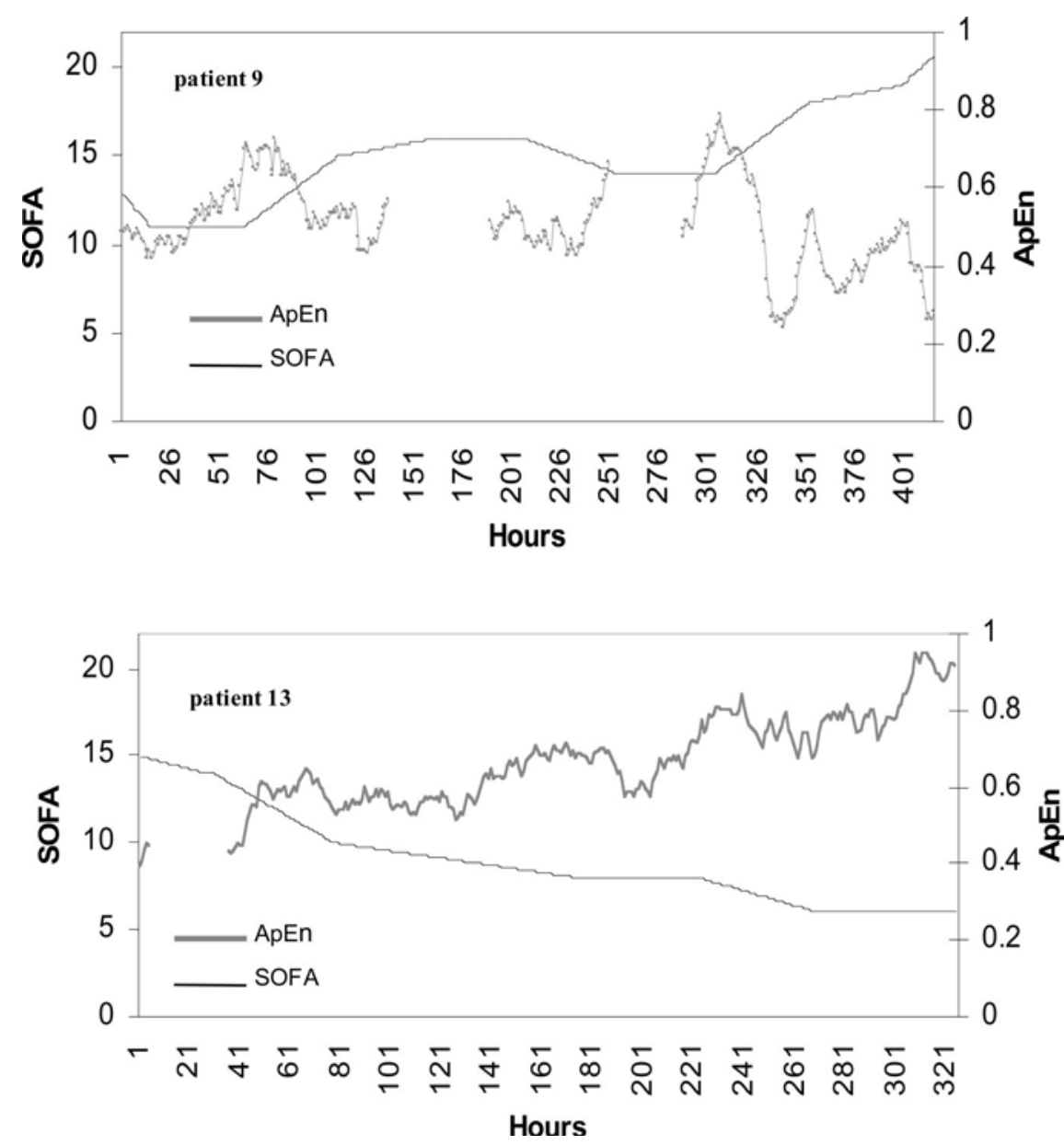

Figure 3. Examples of Sequential Organ Failure Assessment (SOFA) and approximate entropy (ApEn) correlation. Patient 9: 57 yrs old, polytrauma, died. Patient 13: 36 yrs old, multiple-lobar pneumonia, alcoholic deprivation, survived.

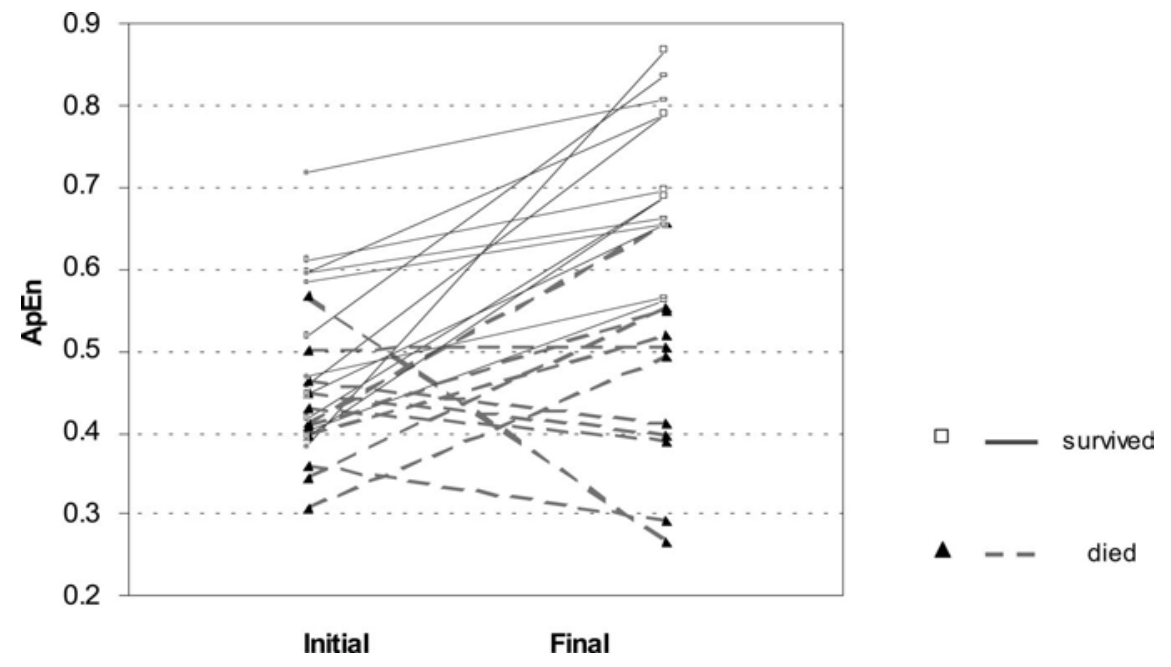

Figure 4. Evolution of approximate entropy $(A p E n)$ in each patient. $A p E n_{(2,0.2 S D, 180)}$ of the initial and final 30 hrs of each patient is displayed. Apen was consistently higher in surviving patients.

status. Initially, we compared the hourly ApEn measures with the latest SOFA score. This would be the "best guess" that the attending physician would have about his or her patient clinical status on real time. However, a posteriori, we had all the SOFA points, so we could get an even better fit interpolating between adjacent SOFA scores. The differences between these two approaches were insignificant. Using the empirical SOFA value (which was considered constant until the next measurement), the average correlation coefficient between SOFA and ApEn was -0.49 (sD 0.26). With the attributed SOFA, the average correlation coefficient was -0.48 (SD 0.29). We finally decided to use the interpolated data because this was the best "clinical portrait" obtained from the patient with conventional tools and we thought ApEn should be correlated with the most accurate evaluation available.

As in an earlier study (2), complexity decreased with age. However, this did not account for differences in survival. ApEn was responsible for larger differences than age in changes in deviance of the logistic regression models fitted, and it remained statistically significant both in the logistic regression and in the analysis of variance results even after adjustment for the effect of age. Thus, although ApEn had an effect independently of age, we chose not to use age-corrected ApEn in our analyses, because from the clinician's point of view, ApEn uncorrected for age effects is more clinically relevant, has a greater statistical significance, and is easier to use.

Survivors and nonsurvivors also differed in the mean and SD of the temperature series (lower mean and greater SD in patients dying). Probably this is just another consequence of the same physiopathologic mechanism underlying the loss of complexity, namely an impairment of thermoregulation. Nevertheless, complexity analysis is arguably a more reliable tool than mean temperature or SD: It reflects more directly the underlying dysfunction and has a greater discriminating power (e.g., a logistic regression model combining both mean temperature and SD would correctly classify $71 \%$ of cases vs. $83 \%$ and $88 \%$ for minimum and mean ApEn, respectively).

The inverse correlation between the severity of the patient's condition and complexity of the temperature curve is not unexpected. Even though health is commonly thought of as a stable and regular situation, whereas disease would be characterized by disorder and pattern disruption, complex physiologic systems actually tend to behave in an opposite fashion $(14-16)$. Thus, healthy output is normally irregular and apparently ran- 


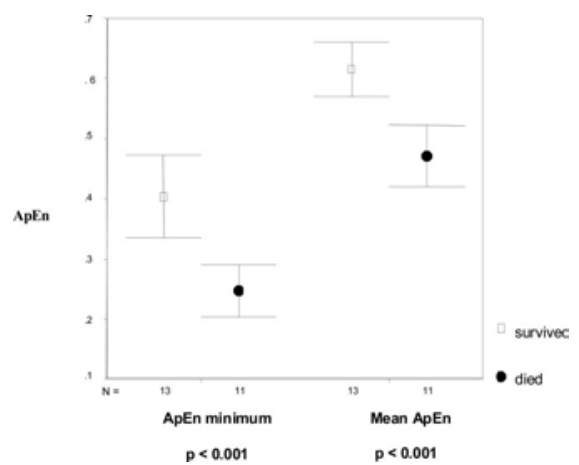

Figure 5. Differences in minimum and mean approximate entropy (ApEn) between survivors and nonsurvivors. Each bar represents the average (and 95\% confidence interval) of the mean ApEn and minimum ApEn attained by patients in each group.

dom, whereas during illness the system's output loses complexity and new rhythms or patterns often emerge. For instance, healthy cardiac frequency is irregular, the irregularity decreasing with age and with the effect of certain pathologies (1722); some cardiac, respiratory, and neurologic disorders are associated with the appearance of a new periodic breathing pattern (Cheynes-Stokes respiration) (23); several neurologic conditions are characterized by the appearance of certain rhythms (e.g., familiar tremor, Parkinson's disease, epilepsy); and so on.

As a rule, under normal conditions, most physiologic systems are subject to multiple stimuli and control mechanisms that give rise to a complex, apparently random output. As the system becomes injured, input and processing are decreased or simplified, and thus the output becomes impoverished and more predictable.

These same considerations also apply to the thermoregulatory mechanisms. Irrespective of whether a patient is febrile, it is reasonable to expect his or her thermoregulatory ability to reflect physical condition. Consequently one would expect to see a reduced complexity in the temperature readings of critically ill patients, the level of complexity mirroring the patient's clinical evolution. In this respect, the mean ApEn value for the patient series was significantly lower than the mean ApEn for a series of 21 healthy subjects. The mean ApEn in the ICU patient group was 0.549 (range, 0.3090.751 ) compared with 0.687 (range, 0.433 $-0.814)$ in the healthy subject series $(p<$ .001) (M Varela, unpublished data). The inverse correlation between the ApEn val-

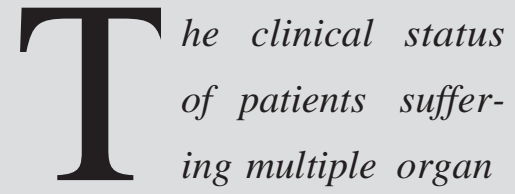

failure is inversely correlated

to the complexity of the tem-

perature curve expressed as

approximate entropy.

ues and the SOFA scores in most of the patients in our series is likewise consistent with this premise.

Measuring the complexity of the temperature curve may offer advantages in clinical settings: It is harmless, noninvasive, and inexpensive, and it allows the patient's physical condition to be read continuously in real time. In contrast, conventional systems like the SOFA score involve relatively invasive and laborintensive analytical determinations that can only be carried out sporadically. Estimates of the complexity of the temperature curve could be of assistance both in assessing functional status and in establishing a prognosis when monitoring critically ill patients.

Finally, complexity analysis could provide a tool enabling us to move beyond the fever/nonfever dichotomy. It could thus make possible a truly quantitative approach to body temperature useful for patients both with and without fever and perhaps hold out applications in other areas of clinical practice.

\section{ACKNOWLEDGMENTS}

We are indebted to Alfredo Martin, Gemma Garcia-Navazo, Goiatz Balciscueta, and Maria Jesus Fernandez-Aceñedo for their help. We are also grateful to Isabel Millan for her biostatistical work and supporting letter.

\section{REFERENCES}

1. Mackowiak PA, Wasserman SS, Levine MM: A critical appraisal of $98.6^{\circ} \mathrm{F}$, the upper limit of the normal body temperature, and other legacies of Carl Reinhold August Wunderlich. JAMA 1992; 268:1578-1580

2. Varela M, Jimenez L, Fariña R: Complexity analysis of the temperature curve: New information from body temperature. Eur J Appl Physiol 2003; 89:230-237
3. Pincus SM: Approximate entropy as a measure of system complexity. Proc Natl Acad Sci U S A 1991; 88:2297-2301

4. Pincus SM, Gladstone IM, Ehrenkranz RA: A regularity statistic for medical data analysis. J Clin Monit 1991; 7:335-345

5. Pincus SM, Goldberger AL: Physiological time-series analysis: What does regularity quantify? Am J Physiol 1994; 266: H1643-H1656

6. Moody GB, Peng CK, Stanley HE, et al: PhysioBank, PhysioToolkit, and Physionet: Components of a new research resource for complex physiologic signals. Circulation 2000; 101:e215- e220. Available at: http://circ.ahajournals.org/cgi/content/full/101/23/e215]. Accessed June 13, 2000

7. Pincus SM: Assessing serial irregularity and its implications for health. Ann N Y Acad Sci 2001; 954:245-67

8. Working Group on Blood Pressure and Heart Variability. European Society of Hypertension. Available at: http://www.cbi.dongnocchi.it/glossary/Home.html

9. Vincent JL, Moreno E, Takala J, et al: The SOFA (Sepsis-related Organ Failure Assessment) score to describe organ dysfunction/ failure. Intensive Care Med 1996; 22:707-710

10. Vincent JL, Mendoça A, Cantraine F, et al: Use of the SOFA score to assess the incidence of organ dysfunction/failure in intensive care units: Results of a multicenter, prospective study. Working group on "sepsis related problems" of the European Society of Intensive Care Medicine. Crit Care Med 1998; 26 : 1793-1800

11. Lopes Ferreira F, Peres Bota D, Bross A, et al: Serial evaluation of the SOFA score to predict outcome in critically ill patients. JAMA 2001; 286:1754-1758

12. Peres Bota D, Melot C, Lopes Ferreira F, et al: The Multiple Organ Dysfunction Score (MODS) versus the Sequential Organ Failure Assessment (SOFA) score in outcome prediction. Intensive Care Med 2002; 28: 1619-1624

13. Timsit JF, Fosse JP, Troché G, et al: OUTCOMEREA Study Group: Calibration and discrimination by daily Logistic Organ Dysfunction scoring comparatively with daily Sequential Organ Failure Assessment scoring for predicting hospital mortality in critically ill patients. Crit Care Med 2002; 30: 2002-2013

14. Goldberger AL, Amaral LAN, Hausdorff JM, et al: Fractal dynamics in physiology: Alterations with disease and aging. Proc Natl Acad Sci U S A 2002; 99(Suppl 1):2466-2472

15. Lipsitz LA, Goldberger AL: Loss of "complexity" and aging. Potential applications of fractals and chaos theory to senescence. JAMA 1992; 267:1806-1809

16. Goldberger AL: Fractal variability versus pathologic periodicity: Complexity loss and stereotypy in disease. Perspect Biol Med 1997; 40:543-561

17. Iyengar N, Peng CK, Morin R, et al: Age related alterations in fractal scaling of car- 
diac interbeat interval dynamics. Am $J$ Physiol 1996; 271:R1078-R1084

18. Pikkujämsä SM, Mäkikallio TH, Sourander LB, et al: Cardiac interbeat interval dynamics from childhood to senescence. Comparison of conventional and new measures based on fractals and chaos theory. Circulation 1999; 100:393-399

19. Huikuri HV, Mäkikallio TH, Airaksinen J, et al: Power-law relationship of heart rate vari- ability as a predictor of mortality in the elderly. Circulation 1998; 97:2031-2036

20. Mäkikallio TH, Hoiber S, Kober L, et al: Fractal analysis of heart rate dynamics as a predictor of mortality in patients with depressed left ventricular function after acute myocardial infarction. Am J Cardiol 1999; 83:836-839

21. Mäkikallio TH, Huikuri HV, Hintze U, et al: Fractal analysis and time- and frequencydomain measures of heart rate variability as predictors of mortality in patients with heart failure. Am J Cardiol 2001; 87:178-182

22. Peng CK, Havlin S, Hausdorff JM, et al: Frac tal mechanisms and heart rate dynamics. Long-range correlations and their breakdown with disease. J Electrocardiol 1995; 28(Suppl):59-65

23. Javaheri S: A mechanism of central sleep apnea in patients with heart failure. $N$ Engl J Med 1999; 341:949-954 